\title{
ENHANCEMENT OF TCP FAIRNESS IN IEEE 802.11 NETWORKS
}

\author{
Bhuvana $\mathrm{LN}^{1}$, Divya $\mathrm{PD}^{2}$ and Divya $\mathrm{A}^{3}$ \\ Department of Information Technology, Easwari Engineering College, \\ Affiliated to Anna University, Chennai \\ ${ }^{1}$ bhuvana.n21@gmail. com \\ ${ }^{2}$ div.pd91@gmail. com \\ ${ }^{3}$ divi_cool8@yahoo.com
}

\begin{abstract}
The usage of fixed buffers in 802.11 networks has a number of disadvantages associated with it. This includes high delay, reduced throughput and inefficient channel utilisation. To overcome this, a dynamic buffer sizing algorithm, the A* algorithm has been implemented at the access point. In this algorithm buffer size is dynamically adjusted depending upon the current channel conditions and hence delay is reduced and the throughput is maintained. But in 802.11 networks with DCF collision avoidance mechanism, it creates significant amount of unfairness between the upstream and downstream TCP flows, with clusters of upstream ACKs blocking downstream data at the access point. Thus a variation of the Explicit Window Adaptation (EWA) scheme has been used to regulate the queuing time of the upload clients by calculating the feedback value at the access point. This creates fairness and increases the number of transmission opportunities for the downstream traffic.
\end{abstract}

\section{KEYWORDS}

Wireless LANs(WLANs), Medium access control (MAC), Transmission control protocol (TCP), Dynamic Buffer sizing, Explicit Window Adaptation(EWA).

\section{INTRODUCTION}

In computer networks, buffers are generally used to store short term packet bursts thereby reducing packet drops and maintaining high link efficiency. Packets are also queued in buffers when the network device lacks the ability to process all the packets immediately.

There are two queuing disciplines namely First In First Out/First Come First Serve (FIFO/FCFS) that can be used by the buffer to queue the packets. In FIFO, the first packet that arrives at the buffer of a network device say for example access point, will be the first packet to be transmitted. When the number of packets exceeds the buffer size the packet at the tail is dropped. This dropping policy is called the tail drop policy. The main disadvantage of FIFO is that it does not discriminate between different traffic sources. It thus led to the introduction of Fair Queuing technique. In this a separate queue is maintained for each flow currently handled by the network device. The network device services these queues in a round robin fashion. Overflowing packets belonging to each queue are discarded. An enhancement of Fair Queuing technique is the Weighted Fair Queue, which assigns a weight to each queue serviced by the network device. The weight defines the number of bits to transmit each time the device services the queue which in turn affects the percentage of links bandwidth the queue will get. The general rule for sizing 
buffers is to set the buffer size to be the product of bandwidth and delay of the flows utilizing the link. This rule is known as the Bandwidth-Delay Product (BDP) rule.

When fixed buffers are used in 802.11 networks it leads to undesirable channel utilization and high delay. The use of dynamic buffer increases the throughput and reduces the delay. In contrast to wired networks the transmission in wireless networks are broadcast in nature. Thus the buffering requirement at each station depends upon the number of active station in wireless LAN. In dynamic buffer sizing techniques the size of buffers is changed to adapt to the changing conditions of the network.

The $\mathrm{A}^{*}$ algorithm which is a combination of eBDP and Adaptive Limit Tuning Feedback algorithms (covered in the later sections) is used to change the size of buffers dynamically. While the $\mathrm{A}^{*}$ algorithm decreases delay and maintains the throughput as achieved by the use of fixed buffers there is a disadvantage associated with this algorithm. The $\mathrm{A}^{*}$ algorithm creates significant amount of unfairness between the upstream and downstream TCP flows. The upstream flow gets a greater share of the links available bandwidth when compared to the downstream flow which gets a very small percentage of the total available bandwidth.

In our project a variation of the Explicit Window Adaptation (EWA) scheme which modifies the Advertised Receiver Window field value of ACK packets at the Access Point is used to create fairness among the different flows.

\section{IEEE 802.11 MAC ALGORITHM}

The Medium Access Control (MAC) Algorithm used by the 802.11 networks is the Distributed Coordination Function (DCF) which is a CSMA/CA based algorithm. According to DCF, when the wireless medium is idle for DIFS (Distributed Inter Frame Spacing) time period, each station initializes a back off counter to a random number selected uniformly between 0 and CW-1 where $\mathrm{CW}$ is the Contention Window size. The time period is slotted and the back off counter is decremented each slot the medium is idle. If the medium is detected as busy the countdown halts and the countdown resumes only when the medium is idle again for DIFS time period. When the counter reaches zero a station transmits packet. If collision occurs the size of Contention Window is doubled and the process gets repeated. When the transmission is successful $\mathrm{CW}$ is reduced to $\mathrm{CW}_{\text {min }}$ and a new countdown is started.

\section{EMUlating BDP ALgORITHM}

In wireless network a fixed value of BDP does not exist because the mean service time is time varying in nature. The service time of each station is measured by observing the time between the arrival of the packet at the network interface queue $t_{s}$ and the successful transmission of packet $t_{e}$. On averaging the packet service time of all stations the mean packet service time $\mathrm{T}_{\text {serv }}$ is obtained.

The eBDP algorithm works as follows. The maximum queuing delay $T_{\max }$ is defined. $1 / \mathrm{T}_{\text {serv }}$ indicates the mean service rate. The buffer size is given as $\mathrm{Q}_{\mathrm{eBDP}}=\min \left(\mathrm{T}_{\max } / \mathrm{T}_{\text {serv }}, \mathrm{Q}^{\mathrm{eBDP}}{ }_{\max }\right)$ where $\mathrm{Q}^{\mathrm{eBDP}}{ }_{\max }$ is the maximum buffer size. For every packet that arrives into the queue the value of $\mathrm{Q}_{\mathrm{eBDP}}$ is calculated. If the queue occupancy is less than $\mathrm{Q}_{\mathrm{eBDP}}$ the packet is put into queue else it is dropped. For every outgoing packet the end service time $t_{\mathrm{e}}$ is recorded as the time at which the MAC ACK arrives for the packet and the service time for each packet is calculated as $T_{\text {serv }}=(1-$ $\mathrm{W}) \mathrm{T}_{\text {serv }}+\mathrm{W}\left(\mathrm{t}_{\mathrm{e}}-\mathrm{t}_{\mathrm{s}}\right)$ where $\mathrm{W}$ is the averaging parameter.

\section{Adaptive Limit Tuning Feedback Algorithm}

To maintain high link efficiency a station must have a packet to transmit whenever it wins a transmission opportunity. Thus the time for which the station buffer is empty should be minimised which can be achieved by maintaining the buffer size sufficiently large. But the use of large buffers can lead to high queuing delays. Thus to ensure low delay the buffer size should be 
as small as possible. Thus the smallest buffer size that ensure high link utilisation should be selected for operation. The following approach is used to ensure effective link utilisation. If the buffer rarely empties the buffer size is decreased. On the contrary if the buffer remains empty for a long period the buffer size is increased.

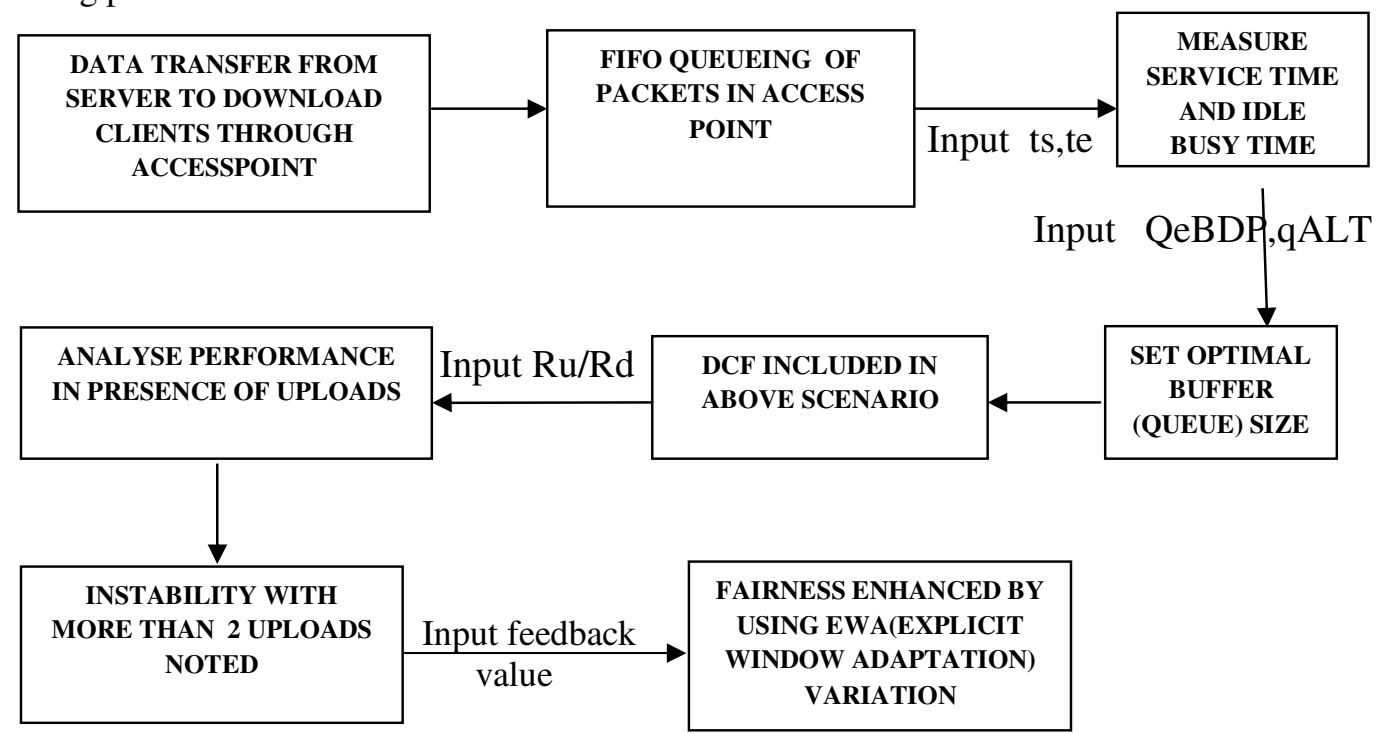

Figure 1: The above figure shows the overall functioning of the wireless network system.

In this algorithm a queue occupancy threshold $\mathrm{q}_{\mathrm{thr}}$ is defined. The amount of time the queue spends below this threshold and amount of time the queue spends above this threshold are observed as $t_{i}(k)$ and $t_{b}(k)$ respectively where $t_{i}(k)$ indicates the idle period and $t_{b}(k)$ the busy period. The time period $t=t_{i}(k)+t_{b}(k)$. The minimum link utilisation is given by $t_{b} /\left(t_{b}+t_{i}\right)$ and $q(k)$ denotes the buffer size at the $\mathrm{k}^{\text {th }}$ observation interval. The buffer size is updated as $\mathrm{q}(\mathrm{k}+1)=\mathrm{q}(\mathrm{k})+\mathrm{a}_{1} \mathrm{t}_{\mathrm{i}}(\mathrm{k})-\mathrm{b}_{1} \mathrm{t}_{\mathrm{b}}(\mathrm{k})$ where $\mathrm{a}_{1}$ and $\mathrm{b}_{1}$ are the design parameters. If $\mathrm{a}_{1} \mathrm{t}_{\mathrm{i}}(\mathrm{k})=\mathrm{b}_{1} \mathrm{t}_{\mathrm{b}}(\mathrm{k})$ the buffer size remains unchanged, if the idle time is larger i.e. $a_{1} t_{i}(k)>b_{1} t_{b}(k)$ the buffer size is increased and if the busy time is larger i.e. $a_{1} t_{i}(k)<b_{1} t_{i}(k)$ the buffer size is decreased. The maximum buffer size is denoted as $\mathrm{q}_{\max }$ and the minimum buffer size is given as $\mathrm{q}_{\min }$. For every time period the idle period is calculated and $\mathrm{q}_{\mathrm{ALT}}$ is measured as $\mathrm{q}_{\mathrm{ALT}}+\mathrm{a}_{1} \mathrm{t}_{\mathrm{i}}-\mathrm{b}_{1}\left(\mathrm{t}-\mathrm{t}_{\mathrm{i}}\right)$ and this value is updated as $\mathrm{q}_{\mathrm{ALT}}=$ $\min \left(\max \left(\mathrm{q}_{\mathrm{ALT}}, \mathrm{q}_{\min }\right), \mathrm{q}_{\max }\right)$.

\section{The A* Algorithm}

The $A^{*}$ algorithm is a hybrid algorithm that uses the mean service time to calculate $Q_{\mathrm{eBDP}}$ in the eBDP algorithm and uses the idle/busy time to calculate the value of $\mathrm{q}_{\mathrm{ALT}}$ in ALT algorithm and sets the buffer size at access point as $\min \left(\mathrm{Q}_{\mathrm{eBDP}}, \mathrm{q}_{\mathrm{ALT}}\right)$. When the channel condition changes the service time measured from eBDP algorithm is used to adjust the buffer size accordingly. It then uses the ALT algorithm to set the buffer size more accurately. 


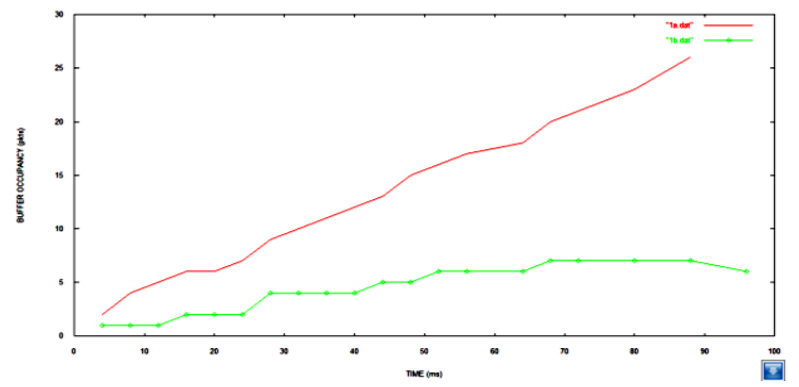

Fixed Buffer

A* Algorithm

Figure2: Illustrates simulation graph obtained for Buffer Occupancy (pkts) vs Buffer Size (pkts) for 5 downloads in case of fixed buffers and $\mathrm{A}^{*}$ algorithm.

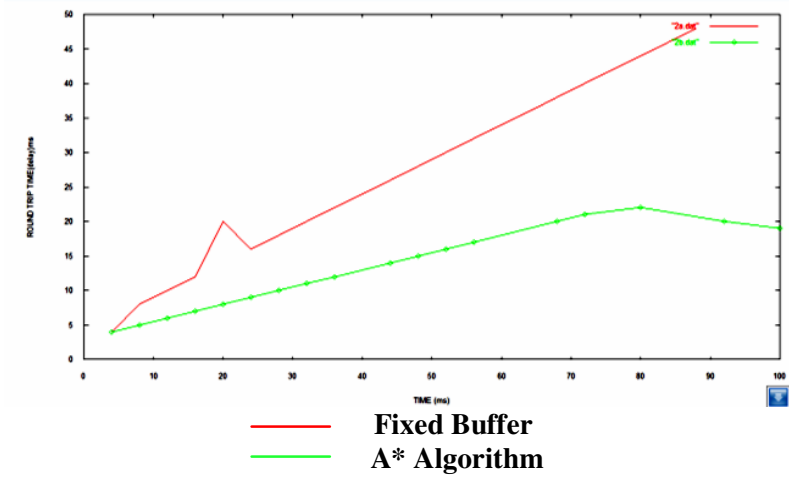

Figure3: Illustrates simulation graph obtained for Delay(RTT) (ms) vs Buffer Size (pkts) for 5 downloads in case of fixed buffers and $\mathrm{A}^{*}$ algorithm.

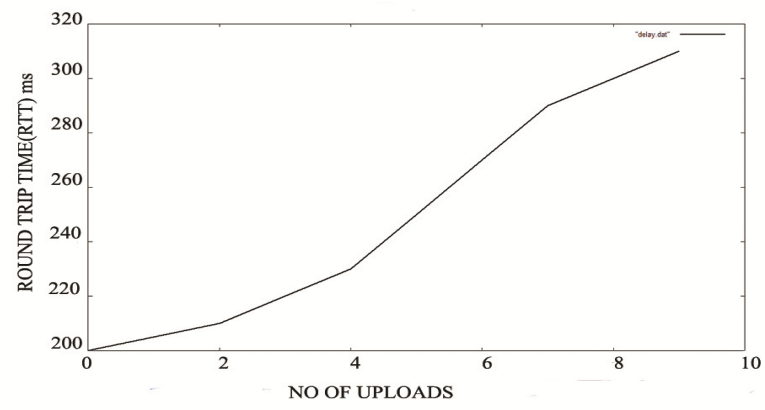

Figure 4: Represents RTT (ms) vs Number of Uploads with A* algorithm.

\section{UNFAIRNESS BETWEEN UPSTREAM AND DOWNSTREAM TCP FLOWS}

Consider a WLAN networks made up of $\mathrm{n}$ client stations. The client stations carry one upload flow each. The access point of the WLAN is responsible for transmitting the ACK packets back to the client. But these ACK may be dropped because of the equal access nature of WLAN, since the data packets get $n /(n+1)$ share of transmission opportunities whereas the ACK packets that 
represent the downstream traffic gets only $1 /(n+1)$ share. This creates significant amount of unfairness between the upstream and downstream TCP flows.

\section{UNDERSTANDING TCP FAIRNESS IN 802.11 NETWORK}

In WLANs the mobile host (sender/receiver) access the network through the access point (AP). IEEE 802.11 ensures equal access to the media for all host.

If there is one sender and remaining are receivers, the access point and sender gets equal access to the medium and i.e. the sender gets half of the total available bandwidth and the remaining half of the bandwidth is used by the access point. The bandwidth available for the access point is shared equally by all receivers.

The equal access nature of medium access protocol results in unfairness. The throughput ratio is given as the ratio between average TCP uplink throughput $\left(\mathrm{R}_{\mathrm{u}}\right)$ and the average TCP downlink throughput $\left(\mathrm{R}_{\mathrm{d}}\right)$. Even when there is a single mobile sender(upstream flow) and a single mobile receiver(downstream flow) the throughput ratio is 1.44 which indicates that the sender receives 1.44 times the receiver's bandwidth.

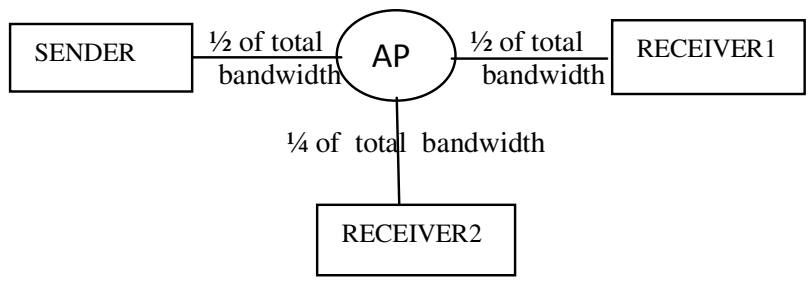

Figure 5: Represents a network made up of one sender and two receivers.

The sender and the access point gets equal share of the channels available bandwidth.

Four regions of TCP unfairness based upon the buffer size at the Access Point are identified. In the first region the buffer size at AP is greater than 84 packets and the throughput ratio is one. This indicates both the upstream and downstream flow gets equal share of the available bandwidth. This is because the buffer is large enough to accommodate the maximum receiver window of both flows. In the second region the buffer size is between 42 and 84 and the throughput ratio lies between 10 and 1 . The third region corresponds to that region where the access point buffer size lies between 6 and 42 and the throughput ratio varies between 9 and 12 . In the fourth region the buffer size is less than 6 .

Case 1 : One upstream and several downstream flows

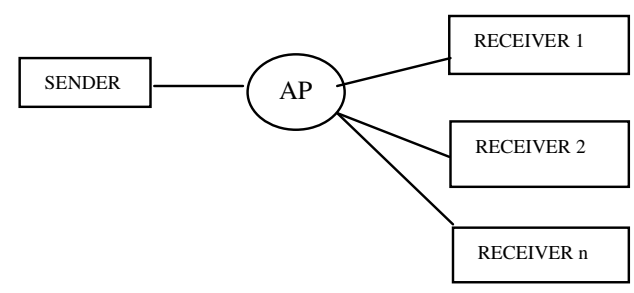

Figure 6: Shows One upstream and $\mathrm{n}$ downstream flows

In this case the throughput ratio is linear i.e. all the downstream flow share the same resources while the throughput remains stable.

Case 2 : Equal number of upstream and downstream flows 


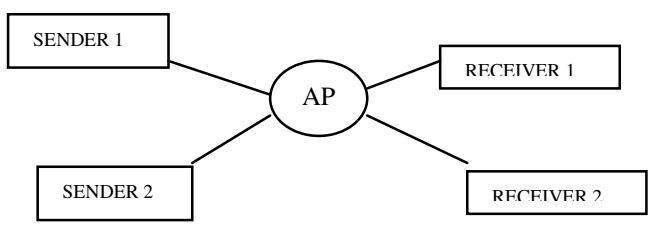

Figure 7: Shows two upstream and two downstream flows

In this case the throughput ratio increases because ACK of upstream flow is cluttered at the buffer of ACK thus the packets of downstream flow experience significant timeout due to packet drop at the access point buffer thus increasing unfairness.

Case 3 : One upstream and one downstream flow

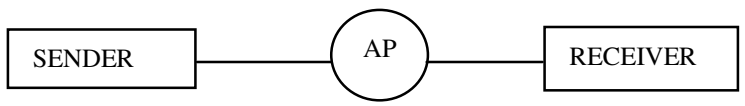

Figure 8: Shows one upstream and downstream flow

In this case the behaviour depends upon the buffer size B at Access Point and the TCP receiver window size (w). When this window size is large enough the loss of ACK packet has no influence on the window size of the sender. This is due to the cumulative acknowledgement nature of TCP in which the next ACK packet will have the appropriate sequence number thereby compensating the loss of previous ACK. The upstream window size increases until it reaches $\mathrm{w}$ and remains the same throughout the period of connection. The downstream TCP window size depends upon B and w. Generally TCP reacts to loss of data packet by halving its window size. Thus if the buffer size at AP is larger than twice the receiver window size al packets will have space in the buffer and packets will not be dropped. Fair allocation of bandwidth to the 3 stations i.e. sender, receiver and access Point by the wireless MAC results in fair allocation of bandwidth to both the TCP flows.

\section{Performance Of A* Algorithm With DCF}

The network scenario we deal with is the one having equal number of senders(upload clients) and receivers(download clients) connected to data source or server through the access point. In this network, when evaluating the performance of the above stated $\mathrm{A}^{*}$ algorithm at access point in the presence of DCF, the system becomes unstable with more than two uploads.

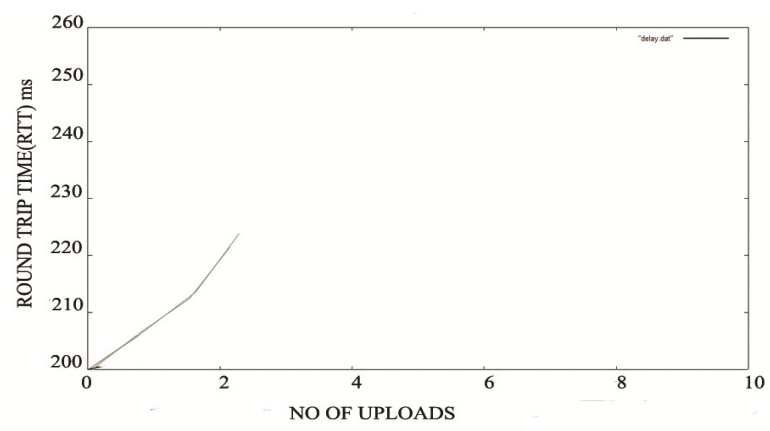

Figure 9: Shows RTT (in ms) vs number of uploads with A* algorithm when DCF mechanism is applied.

This is due to the combined delay caused by DCF back off interval and packet loss due to eBDP. 


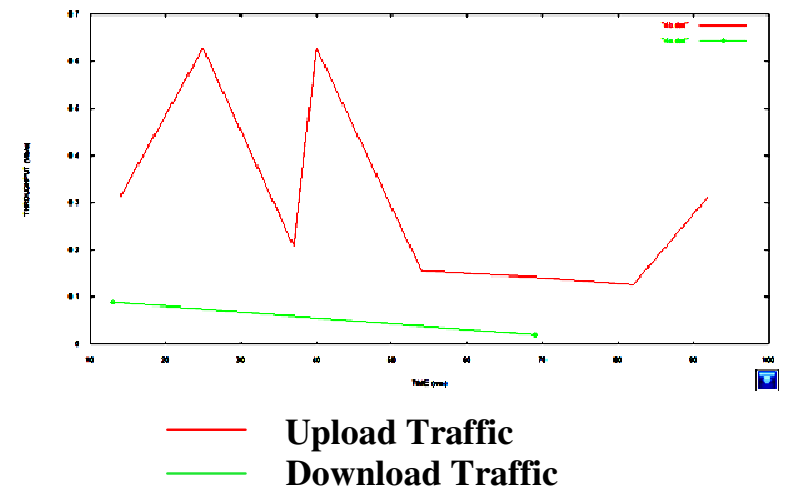

Figure 10: Shows the simulated values of Throughput(Mb/s) vs Time(ms) for Uploads and Downloads in above stated network scenario.

\section{Explicit Window AdAptation}

In this scheme, the advertised receiver window field present in the ACK packets from the data source are used. This field is used to indicate the amount of space available at the receiver. Lowering the value of this field in the access point can help in notifying the upload client that it should reduce the amount of data it sends to the receiver.

\subsection{Functioning of EWA}

The EWA works as follows. An explicit feedback value is sent to the upload client. This feedback is present in the advertised receiver window field of the ACK packets. This feedback is given as a function of the amount of free space in the access point buffer.

At the access point, if the value in the current advertised receiver window field value $W(t)$ is greater than the calculated feedback value $f\left(B_{e}(t)\right)$, the value in the advertised field is reduced to the feedback value. The amount of free space in buffer $B_{e}(t)$ is calculated as $B_{e}(t)=B-Q(t)$ where $B$ indicates the total buffer size and $Q(t)$ the buffer occupancy at time $t$. Thus the final feedback value $\mathrm{W}_{\mathrm{r}}{ }^{\circ}(\mathrm{t})$ is calculated as a function of the amount of free buffer space at the access point i.e. $\mathrm{f}\left(\mathrm{B}_{\mathrm{e}}(\mathrm{t})\right)$ is $\mathrm{W}_{\mathrm{r}}^{\prime}(\mathrm{t})=\min \left(\mathrm{W}_{\mathrm{r}}(\mathrm{t}), \max \left(\mathrm{f}\left(\mathrm{B}_{\mathrm{e}}(\mathrm{t})\right)\right.\right.$, MSS $\left.)\right)$, where MSS is Maximum Segment Size.

\section{Variation Of EWA To Create Fairness}

Once the modified advertised window field is detected by the upload client, it increases its queuing time to increase the number of opportunities for the downstream traffic. When the queuing time is increased, the downstream ACKs get opportunities to return to upload clients and hence their clutter at the access point is reduced. This increases the transmission opportunities for download data thus enhancing fairness. 


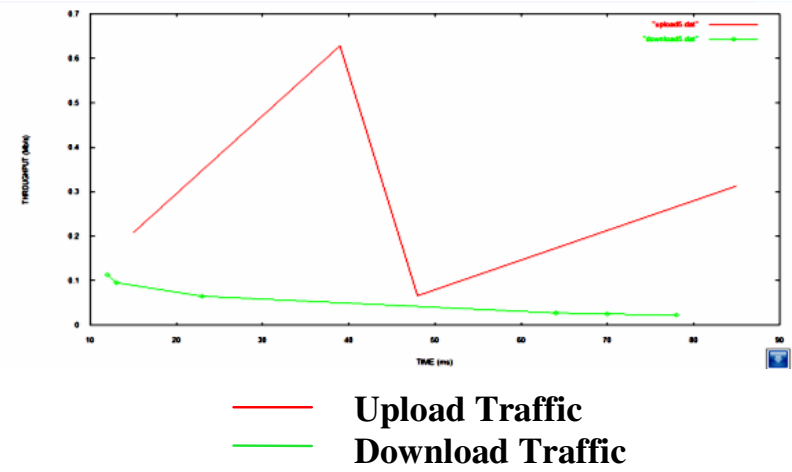

Figure 11: shows the simulated values of Throughput(Mb/s) vs Time(ms) for Uploads and Downloads after EWA variation is implemented in the above network scenario.

\section{CONCLUSION}

Thus by increasing the queuing time in explicit feedback scheme, fairness among the upstream and downstream flows is achieved when using dynamic buffers. This technique also leads to perfect utilization of the channels bandwidth. A major disadvantage in this technique is that it can be applied only to those routers that can manipulate the transport layer.

\section{REFERENCES}

[1] Tianji Li, Douglas Leith and David Malone in "Buffer Sizing for 802.11 Based Networks ", February 2011 .

[2] Lampros Kalampoukas, Anujan Varm and K. K. Ramakrishnan in "Explicit Window Adaptation: A Method to Enhance TCP Performance”, June 2002.

[3] Saar Pilosof, Ramachandran Ramjee, Danny Raz, Yuval Shavitt and Prasun Sinha in "Understanding TCP Fainess Over Wireless Lan'.

[4] D. J. Leith, P. Clifford, D. Malone, and A. Ng in "TCP Fairness in 802.11e WLANs", November 2005.

[5] Tianji Li and Douglas J. Leith in "Buffer Sizing for TCP Flows in 802.11e WLANs", IEEE communications letters, vol. 12, no. 3, March 2008. 


\section{AUTHORS}

Bhuvana Lalitha $\mathbf{N}$ is a graduate in B.Tech Information Technology from Easwari Engineering College, Chennai, India and is joining Tata Consultancy Services in August 2012 . Her research interests are in the field of Wireless Networks and Robotics. Email: bhuvana.n21@gmail.com

Divya A, a graduate in B.Tech Information Technology from Easwari Engineering College, Chennai, India, campus-selected in Tata Consultancy Services in 2012 . Her research interests are in the field of Telecommunication and Networks.

Email: divi_cool8@yahoo.com

Divya $\mathbf{P} \mathbf{D}$, is a graduate in B.Tech Information Technology from Easwari Engineering College, Chennai, India. She will be joining Tata Consultancy Services shortly. Her research interests are in the field of Networks and Router security. Email: div.pd91@gmail.com
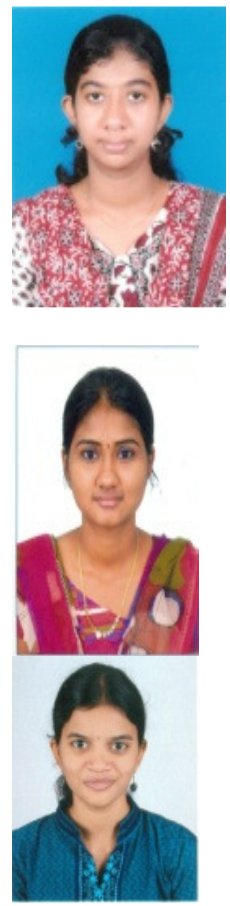